\title{
Artificial Intelligence for Managerial Information Processing and Decision- Making in the Era of Information Overload
}

\author{
Christian Dietzmann \\ Leipzig University, BEI St. Gallen AG \\ christian.dietzmann@bei-sg.ch
}

\author{
Yanqing Duan \\ University of Bedfordshire \\ yanqing.duan@beds.ac.uk
}

\begin{abstract}
In the big data era, managers are exposed to an increasing amount of structured and unstructured information that they must process daily to make decisions. In this context, artificial intelligence (AI) functionalities can support managerial information processing (IP), which forms the basis of managers' decision-making. To date, little is known about the themes that managers face when integrating AI into their IP and decision-making. The present paper identifies these through three focus group interviews with managers from the financial industry, validates them through a survey and derives organizational implications. The results imply that organizations should (1) evaluate managerial IP tasks and matching AI systems, (2) (re)define roles for managers and AI systems, and (3) redesign management processes for sustainable human-AI interaction.
\end{abstract}

\section{Introduction}

The discussion on artificial intelligence (AI) usage often evolves around process optimization or pattern recognition in large datasets. While process optimization is mostly motivated by efficiency gains, the second application field addresses a human problem: The human brain is by far the most efficient computer, but neither processing speed nor storage capacity can be expanded at present. In a world where the amount of digital data generated annually is predicted to increase from 45 zettabytes in 2019 to 175 zettabytes in 2025, humans will very soon reach their brain power limits [1]. Smart devices provide us with all kinds of real-time information and are in competition with our attention in the real world. Anyone who has thought that Elon Musk's idea of the brain-computer interface "Neuralink" to expand information processing (IP) is a marketing move or an AI dystopia will be puzzled by a glance at their own smartphone usage statistics [2].

The relevance of $\mathrm{AI}$ in decision-making is obvious since human intelligence and AI capabilities are assumed to be complementary and beneficiary in combination with each other [3] [4] [5]. Researchers have found that AI may be suitable for unstructured decision-making which is mostly conducted by managers. A study by Parry et al. (2016) discusses scenarios of leadership decision-making support and substitution by AI in non-routine decision processes [6]. On the one hand, it would be advantageous if AI may support managers in pattern recognition to formulate a vision for the organization, mitigate agency problems, de-individualize managerial decision-making and eliminate trust issues. On the other hand, automated leadership decision-making may underweight subjective, non-computable and overweigh objective, computable criteria and has not reached a maturity yet to consider highly social interplays. Also, de-individualization of leadership may be perceived as an imposed rationalization of managerial decision-making by the operational levels. The scientific literature discusses the organizational impacts of AI in decision-making but lacks a detailed understanding of the managerial viewpoint towards AI integration into their IP. Duan et al. (2019) stress the importance of re-defining the AI concept to account for the changing nature of its applications for the analysis of organizational transformations based on changes in decision-making [7]. Krogh (2018) also underlines the importance of investigating organizational impacts of $\mathrm{AI}$ and requirements for organizational design with AI-based decision-making authority [8]. Shrestha et al. (2019), e.g., propose a framework for the design of human-AI decisionmaking structures [9]. Other authors like Seidel et al. (2019) and Zheng et al. (2017) discuss the human-inthe-loop concept evolving around human-AI collaboration [5] [10]. The existing literature has not identified themes that are important to managers in integrating $\mathrm{AI}$ in decision-making. Hence, this article addresses the following research questions (RQ):

$R Q$ 1: What are the relevant themes for AI integration into managerial decision-making?

$R Q$ 2: What organizational implications evolve around the identified themes?

The remainder of the paper is as follows: Chapter 2 contains the theoretical background of IP, information overload and AI-based decision-making. Chapter 3 presents the methodology, and chapter 4 comprises the focus group interview results. Chapter 5 validates these results applying a survey, while chapter 6 discusses the organizational implications and theoretical contributions of the results. Chapter 7 concludes the paper. 


\section{Theoretical background}

\subsection{Information processing}

Managers need information to make informed decisions, but they are facing increasing complexity, diversity and uncertainty in processing information for making decisions [11]. Based on Simon's four decision-making phases 'intelligence', 'design', 'choice' and 'intelligence', IP supports the 'intelligence' phase that involves examining the organizational environment to identify new varieties that call for new actions [12]. Ong et al. (2020) describe IP as (1) the identification and selection of information sources, (2) the articulation of a query, question, or topic, (3) extraction of information, (4) evaluation of retrieved information, (5) filtering for irrelevant information and finally (6) interpretation [13]. Although there are different terms used for IP, so far, the scientific literature offers no clear approaches to define the term 'information processing'. Most of the relevant studies on IP use the terms 'information behavior' or 'information processing behavior'.

According to Bawden and Robinson (2011), information behavior is individual and distinguishes by individual information seeking, scanning, and manipulating activities [14]. Wilson (1999) reviewed and presented an outline of models on information behavior, exploring the relationship and various aspects of information behavior models [15]. Wilson's original and revised model of information behavior shows that the core elements of information behavior are information seeking and processing behavior. Wilson (1981) identified that IP includes the context of information need, personal, role-related, and environmental barriers, and information-seeking behavior like starting, chaining, browsing, differentiating, monitoring, extracting, verifying, and ending [16]. Ellis et al. (1993) further developed Wilson's (1981) information behavior empirically and proposed a sequential model of IP behaviors. In contrast to Wilson, Ellis distinguishes the behaviors between search behavior (starting, chaining, extracting, verifying, ending) and macro information behavior (browsing, monitoring, differentiating) [17]. Furthermore, it can be stated that Kuhlthau's (1993) information search process is in a way an abstraction of Ellis' model, which includes the stages tasks 'initiation', 'selection', 'exploration', 'formulation', 'collection' and 'presentation' [18]. Wilson's 1996 model of information behavior, which is a revised version of the 1981 model, added four types of 'information seeking behavior' [19]. These are 'passive attention', 'passive search', 'active search', and 'ongoing search'. Furthermore, Wilson's new model contains a feedback loop 'information processing and use' from the information seeking stage back to the beginning of the process to determine the success of the information behavior. Spink (2010) states that information behavior is influenced by the individual's cognitive, affective, and societal status [20]. While the previously listed literature focuses on IP behavior, Duan et al. (2012) adapt relevant aspects of the before mentioned IP behavior literature and present a definition of IP in the course of the 'intelligence' phase of Simon's decision-making theory. To derive design criteria for intelligent software agents to support business executives, the authors define and use the terms 'information acquisition', 'information synthesis', and 'information sensemaking' to describe managers' intelligence activities involving IP for decision-making [11].

In summary, the literature review on the relevant studies suggests that IP models can cover a wide range of issues from different perspectives including social and organizational learning, decision-making, psychology, and communication. Based on the review of relevant literature, this paper follows the approach of Duan et al. (2012) and defines IP as consisting of the three interrelated core activities 'information acquisition', 'information synthesis' and 'information sensemaking'. Information acquisition is defined by the present study as information scanning and searching from the information environment, as well as managing a variety of information contents, sources, and formats. Information synthesis is defined as actions to screen out irrelevant information and/or refine information acquired for sensemaking. Information sensemaking, in term, is the process of the construction of meaning and understanding in information for information use. The connections between the core IP activities of the abstracted IP model are bidirectional and inspired by Wilson's 1996 model which includes a feedback loop. Within the abstracted IP model, individuals can go back to acquire more information if they need have more information for sensemaking (Figure 1).

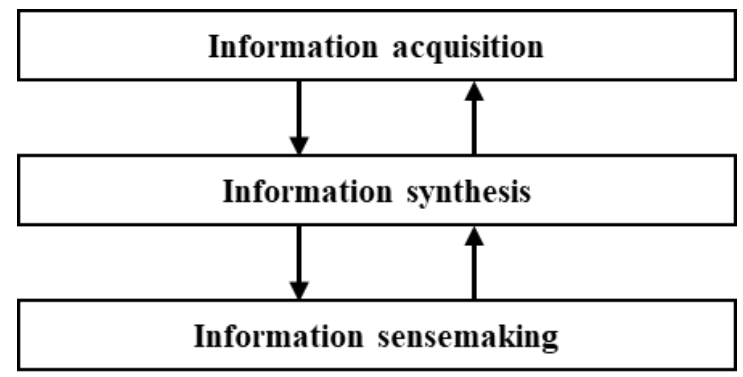

Figure 1. Abstracted information processing model. 


\subsection{Information overload and decision- making}

While Abraham Lincoln walked miles to get a book and consume the information he was interested in, it was a cornerstone of human evolution to establish libraries where a collection of books and hence knowledge was gathered. Nowadays, there is no need for libraries anymore, as most information is available online via PC, smartphone and other communication technologies [21]. Since human mankind has always been fascinated by the constantly increasing amount of available information being produced and to be processed, research on information overload (IO) begins even before the era of big data. Several definitions are assigned to the term 'information overload', but a uniform definition is not yet existing [22]. A scientifically recognized IO approach is the inverted U-curve characterized by a decreasing decision accuracy once a certain point of information load is exceeded [23]. Once the information to be processed exceeds the amount on the basis of which the individual can derive decisions (individual IP capacity), decision makers face IO (Figure 2). Following this view, Schroder (1967) argues that there exist at least two factors that determine the shape of the inverted U-curve [24]. First, a decision maker is confronted with resource-related constraints, that is, limitations resulting from scarce individual resources such as time or velocity [21]. An IO might occur earlier if the decision maker has only limited time in using the available information efficiently. Second, the point at which IO is reached is strongly dependent on informational and human characteristics. Given the processing capacity of a decision maker, informational complexity, the level of redundancy or inconsistency contribute to an early overload. Likewise, given information characteristics, it is to be expected that an individual with only limited short-term memory or processing capacity will be prematurely overloaded [22] [23].

In 1960, Miller (1960) already found that information input overload may impact human cells,

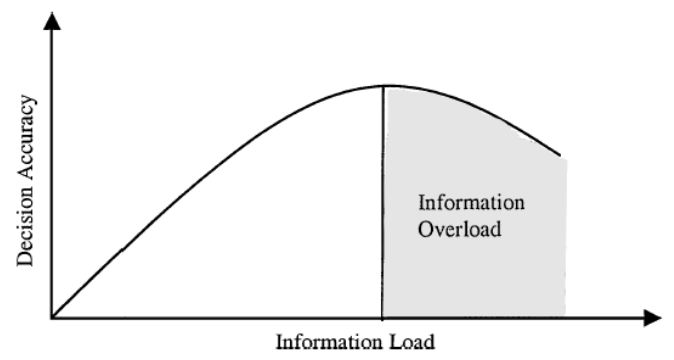

Figure 2. The inverted U-curve of information overload (Eppler \& Mengis, 2004). organs, the individual, groups as well as social institutions [25]. More precisely, IO in social organizations is considered a risk towards its operational performance since it causes stress and exhaustion [26]. In this context, the role, support, and design requirements of management information systems were increasingly discussed. Ackoff (1967) claimed that these systems are actually 'management misinformation systems' since they show major deficiencies. Therefore, he proposed five assumptions for the design of such systems. From his viewpoint, managers (1) lack relevant information, (2) need the information they want, (3) make better decisions with appropriate information at hand, (4) improve organizational performance by increased communication quality and (5) only need to know how to use an information system - not how it works [27]. Nowadays, IO is mainly caused by information systems both in private and at work, but it may also be caused by tasks, environment, personal characteristics, and information source design [22]. Information systems causing IO include email applications, intranet websites and the internet in general [28]. The more responsibility managers hold in an organization, the more they solve novel and unstructured tasks, socalled indeterminate problems. Hence, they practice unprogrammed problem-solving which can be negatively influenced by IO and in term affects decision-making quality [29] [30]. IO makes it difficult for managers to obtain useful and relevant information and therefore generate insights [31]. The insights enable organizations to learn and convert illdefined into well-defined problems (Figure 3). If the starting point, permitted actions and the target state are known, organizations can develop procedures and processes to solve a task repetitively [30]. Decisions made in the strategic apex are affecting the organization in a top-down manner, meaning that according to the organizational structure of [32] [33] the middle line, technostructure, support staff and the operative core are directly or indirectly subject to the quality of managerial decisions.

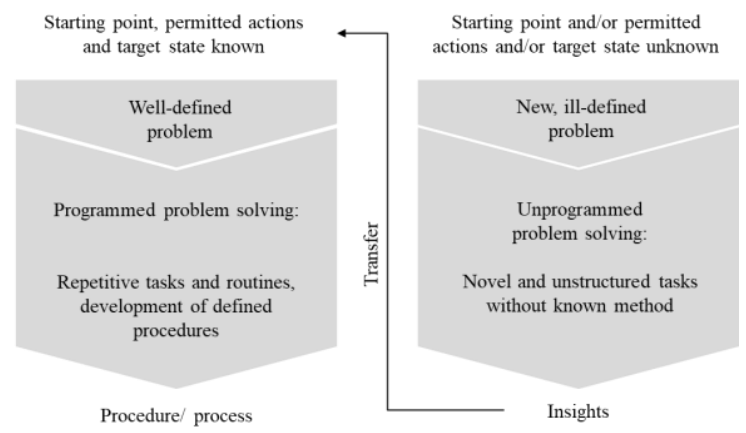

Figure 3. Transformation of ill-defined into welldefined problems. 


\subsection{Artificial Intelligence for managerial decision-making}

According to Herbert Simon (1960), the decisionmaking process consists of the four phases 'intelligence', 'design', 'choice', and 'implementation. While the 'intelligence' phase means identifying problems, 'design' comprises deriving (counter)actions that fit the underlying problem [34]. In the 'choice' phase, one or more appropriate actions are chosen, and the problem is fixed by applying the action in the 'implementation' phase. The managerial decision-making process is influenced by uncertainty, complexity and equivocality of situations offering potential for AI use. While uncertainty in decision-making is characterized by a lack of alternatives and consequences, complexity arises from missing decision-relevant variables and thus a need for superhuman cognitive skills, while equivocality is based on different perceptions of decision situations by involved parties [4]. AI can support managerial decision-making with a broad range of functionalities [35]. To make them comparable to human abilities, the human intelligence abilities of Gardner (1999), Dietzmann and Alt (2020) mapped the AI functionalities along the agent-based AI definition of Russell and Norvig (2016) in a periodic table [36] [37] [38]. The complexity of every single AI functionality contained in the periodic table of AI can be measured by the number of associated human intelligences - the more human intelligences are mapped onto a functionality, the more complex it is to design such an AI-based application. AI functionalities which are easy to perform by humans, such as speech recognition, communication, and mobility, are difficult to conduct by AI applications (intuitive decision-making). The other way around, AI functionalities which can be easily performed by AIbased algorithms, such as synthetic reasoning, explanatory inference, data analytics, control, and category learning are connected to mathematical intelligence and are more difficult to execute by humans (rational decision-making). The comparison of AI functionalities shows that the competencies of humans and AI are largely complementary: While humans are naturally capable of making better intuitive decisions, AI algorithms are well suited for rational decision-making [4] [39]. Therefore, AI can support humans in making better decisions by analyzing large datasets and producing forecasts as well as predictions, e.g., which underlines that humans and AI are suitable for cooperation [3]. While managers can be supported in either perceiving, processing, action, or learning, they can be also supported by a combination of functionalities included in an application. While the perception functionalities of the periodic table of AI except 'text extraction' may be less relevant for managers, the processing functionalities clearly add value within the decisionmaking process. The functionality 'decision-making' should be interpreted as a binary element stating whether an AI-based application is allowed or not to decide on its own. The individual character of every AI functionality or application impacts decisionmaking in various ways depending on the specificity of decision search space, interpretability of the decision-making process and outcome, alternative set size, decision-making speed, and the replicability of outcomes [9]. Since the AI-based and the human decision-making in general and individually vary in terms of the before mentioned conditions, the impact of every single functionality or application on a manager's decision-making differs. The evolving organizational decision-making structures may be (1) full human-to-AI delegation, (2) AI-to-human sequential decision-making, (3) human-to-AI sequential decision-making or (4) aggregated humanAI decision-making [9]. Hence, the way organizational decisions are made will affect vertical and horizontal organization structures [8].

\section{Methodology}

The first step includes three one-hour focus group interviews with 14 executive managers to identify relevant themes for AI integration, which are validated applying a survey in step two.

\subsection{Data collection}

The 14 focus group interviewees are part of a consortium research project with financial industry companies from Switzerland, Germany, and Austria (Table 1). One of the project goals is to investigate the organizational integration of AI systems and the evaluation of their impact. All the focus group interview participants are involved in strategic

Table 1. Focus group sample characteristics.

\begin{tabular}{|c|l|l|}
\hline No. & \multicolumn{1}{|c|}{ Role } & \multicolumn{1}{c|}{ Company } \\
\hline 1 & Senior Banking Architect & IT company \\
\hline 2 & Product Manager Innovation & Stock exchange \\
\hline 3 & Project Manager & Banking-IT service provider \\
\hline 4 & Innovation Manager & IT service provider \\
\hline 5 & Director Omnichannel Management & Cantonal bank \\
\hline 6 & Senior Consultant & Bank consulting company \\
\hline 7 & Lead Innovation Manager & Retail bank \\
\hline 8 & Director Change Management & Private bank \\
\hline 9 & Director Business Development & Retail bank \\
\hline 10 & Director Digitization & Retail bank \\
\hline 11 & Product Manager Innovation & IT company \\
\hline 12 & Chief Architect & IT service provider \\
\hline 13 & CEO & Banking-IT service provider \\
\hline 14 & Manager Digitization & Retail bank \\
\hline
\end{tabular}


decision-making and consist of 11 senior executives and 3 middle executives and include digital transformation managers as well as IT and business architects from eight Swiss and each three Austrian and German financial sector companies. The financial industry is well suited to the study of AI integration for decision-making because of its traditionally high information density and early technology adoption [40]. Prior to the interviews, the questions evolving around the three key IP activities and AI-based decision-making were provided to the participants (Table 2). With the managers' consent, the interviews were recorded and transcribed.

\subsubsection{Data analysis}

The focus group interview analysis follows the thematic qualitative analysis method for deductive and inductive analysis [41]. In the first step, general topics were identified and structured from the raw focus group interview data. In the second step, a detailed interpretive conceptual analysis and mapping of relevant statements was conducted to interpret the meanings of themes and to identify any consensus, dilemmas, and contradictions by reading and rereading quotes from each emerging. Interpretation reliability was assessed through inter-rater reliability analysis, in which two researchers performed double blind interpretation with a view to reaching the intersubjective agreement among interpreters [42]. Meaningful and relevant issues were elicited and coded into themes in first and second order, by referring to the abstracted IP model introduced in chapter 2.1. With the high volume of raw data obtained from all the transcripts, the qualitative analysis software NVivo was selected and applied for efficient handling, managing, searching, displaying, and analyzing of findings.

Table 2. Focus group interview questions.

\begin{tabular}{|l|l|}
\hline $\mathbf{1}$ & \multicolumn{2}{|l|}{ Introductory questions } \\
\hline 1.1 & What are your most important challenges of today's information processing? \\
\hline 1.2 & What are the reasons for these challenges? \\
\hline $\mathbf{2}$ & \multicolumn{1}{|l|}{ Information acquisition } \\
\hline 2.1 & Do you rather obtain your information through information systems or personally? \\
\hline 2.2 & Are Big Data and AI changing the way you obtain information? \\
\hline 2.3 & $\begin{array}{l}\text { Do you rather trust (intelligent) information systems or "knowledge workers" when } \\
\text { gathering information? }\end{array}$ \\
\hline $\mathbf{3}$ & Information synthesis \\
\hline 3.1 & $\begin{array}{l}\text { Do you use analytics/forecasting tools, simulations, or scenario analysis to analyze the } \\
\text { relevance and/or urgency of information? }\end{array}$ \\
\hline 3.2 & $\begin{array}{l}\text { Do you receive too much information so that you must rely on your intuition to analyze the } \\
\text { information? }\end{array}$ \\
\hline $\mathbf{4}$ & Information Sensemaking \\
\hline & $\begin{array}{l}\text { Do you judge information rather on the basis of your experience, knowledge or your } \\
\text { network? Or do you increasingly use intelligent systems/AI applications for this purpose? }\end{array}$ \\
\hline $\mathbf{5}$ & Future role of AI in managerial decision-making \\
\hline & $\begin{array}{l}\text { Which steps of decision-making would you like to support or replace by AI? } \\
\text { a) Information acquisition } \\
\text { b) Information synthesis } \\
\text { c) Information sensemaking }\end{array}$ \\
\hline 5.2 & If an AI system could do everything you want, what roles and tasks would you assign to it? \\
\hline 5.3 & $\begin{array}{l}\text { What are your concerns with AI usage - would you trust an AI-based application for } \\
\text { decision-making support? }\end{array}$ \\
\hline
\end{tabular}

\section{Focus group interview results}

The focus group interviews revealed managerial challenges in IP as well as advantageous and disadvantageous aspects of AI in decision-making.

\subsection{Managerial information processing}

The themes evolving around managerial IP were merely elicited from interview questions 1, 2, 3 and 4 which are especially focusing on this topic. Figure 4 provides a summary of the three identified main themes (1) managerial IP challenges, (2) criteria for information system usage in IP, and (3) technological challenges for information system integration in IP.

The code analysis in terms of (1) managerial challenges in IP revealed that managers suffer from IO especially during information acquisition due to the internet and social media as resources of unlimited information. One manager points out that this makes time for information acquisition scarce by stating that managers "... have less and less time to do a thorough research.” (group 1). Unstructured information and managers' time constraints complicate information scanning and search and hence drive IO: “... it is more like not getting the right information. From my point of view. Too much information ..." (group 2). Another manager underlines the difficulties of managing information contents and sources in the course of information acquisition: "... we get push news at all times, that gets on my nerves, but I want to be able to decide for myself when and where I access any source of information." (group 3). IO also affects the phase of information synthesis which is a key behavior to filter relevant information and transform it into usable information. One participant highlighted, “... that you are overwhelmed by the information and then you have to search for what you really need [...] I always find that relatively difficult." (group 3). Another manager also describes the importance of information synthesis in terms of information relevance and objectivity: "What is coming in the next months and years? [...] there are so many different directions, and you have to filter well." (group 3). Furthermore, the managers communicated a lack of tools that support IP. One reason is a lack of organizational strategies for tool-based IP support and another one is data protection regulations. One manager stated that "We are not really ready yet, we are trying to get a strategy for this topic. In which direction do we want to go with it? That means we are still far away from concrete tools." (group 1). Another participant is of the opinion that "... if we want to offer person-specific services that are AI-supported, then we will again run into the old problem of identifying the individual person. It 


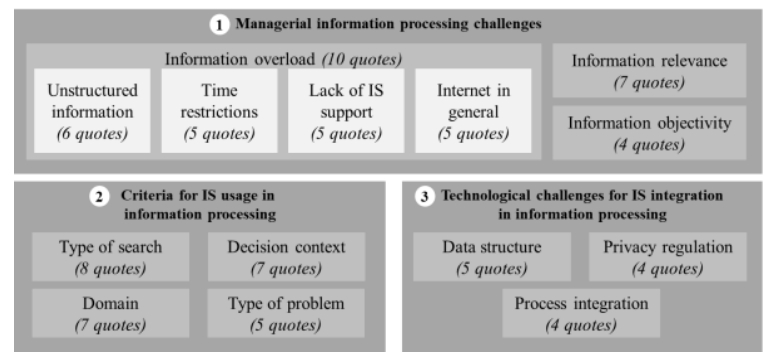

Figure 4. Identified themes and number of quotes on managerial information processing

prevents the assignability of a person. On the one hand it is positive, because of data protection, but on the other hand it prevents qualitatively better results ..." (group 1). As a result of the before mentioned limitations, managers are in turn facing difficulties in information acquisition: "I think the life of information is also getting shorter and shorter. Therefore, it is very important to check things against reality. Because what was valuable yesterday may not be valuable tomorrow." (group 2).

In terms of (2) criteria for information system usage, the code analysis resulted in four decisionmaking-related criteria. According to the managers, the IS usage depends on the search type, decision context, domain, and type of problem. According to one participant, an IS-based search "... is always worth a short, active search for information, for basics ..." (group 1). Another interviewee compares ISbased search with a conversation: "If you then make a targeted search, you also go into conversations, just as you do with the media or with the web or with an information base, exactly the same. You go to people who you think can say something about this topic." (group 1). This statement underlines that IS usage is very common in managerial IP and that they are not permanently relying on human assistance but understand IS systems as a natural source of information. If IS support makes sense in managerial decision-making also depends on the decision context: "I must make a decision as to whether to grant a loan, so I work with customer data [...]. If, on the other hand, you decide on the bank's general strategy for the next ten years, then you base your decision less on data and more on the experience you gain in your interaction with other people." (group 1). The domain of IP also plays a major role if a manager needs support by an IS: "I think it is significantly different whether you work in the service sector for banks [...] or whether you work in the bank itself." Besides of that, IS support for IP is difficult to implement in certain organizational functions: "Well that's a special case because I'm working in the field of innovation." (group 1). Often, people rely on "reputation or the doctorate" (group 3) when it comes to the decision between choosing a human or IS. Finally, the problem type influences the need and sense of IS support in IP: "It always depends extremely on whether there is an explicit answer to what I am looking for. Often, I have a rough thought or a rough idea about what I want information about, and then I guide myself over the net or over books. " (group 3).

Regarding (3) technological challenges for information system integration in IP, four main challenges are identified. Besides data structure, privacy regulation, and a lack of process integration, the low degree of automation within organizations are challenges for IS integration in managerial IP. One participant offered a vivid example for a data migration challenge: "First of all, the customer is on our homepage, as an anonymous customer, looking at the offers, we can see that, but we do not know who it is. Only when he has logged in do we know that it is Mr. Müller, that it is Mr. Meier, etc. And that is the challenge, to consolidate these two databases and then know that he or she was looking for construction financing." (group 1). Managers are also complaining about raw, unprocessed data. Another challenge lies in privacy regulations: "We are building up a data lake where we also merge [data], and above all, it is difficult to have two separate spheres due to data protection regulations." (group 1). A lack of process integration is another challenge for IS integration in managerial IP: "The idea is there, the theory is there, the potential is recognized, but it is not integrated into the processes ..." (group 1).

\subsection{Artificial intelligence for managerial decision-making}

The four main themes on AI for managerial decision-making are not only extracted from question 5 , but also from questions 1 to 4 since all three focus groups already addressed AI and decision-making earlier in their discussions. During the code analysis, four main themes around AI and managerial decisionmaking were identified (Figure 5). First, the participants communicated that they view the database as an important criterium for AI usage in decisionmaking: "I would say that in areas where we actually have a solid database that makes something like [AI] possible, this is not used in our bank, even though we have the basis." Next, database validity is important to the managers: "Is the data actually available, and correct?" (group 2). Additionally, the participants would use AI for decision-making if they had the opportunity of human validation either by themselves or by an assistant. The use of AI-based decisionsupport also depends on internal or external expert availability, and if the task frequency is high. One 


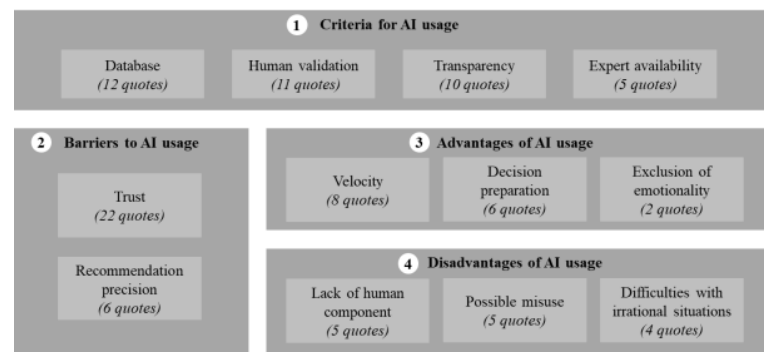

Figure 5. Identified themes and number of quotes on Al for managerial decision-making.

participant states that "... it is not an 'either or' but an 'both and' [between AI-based IS and humans]. And as time goes on, it will tend to move towards analysis systems, because more and more things can be evaluated using data systems. But I think the hybrid model is important here." (group 2). The aspect of human validation is strongly connected to the question whether managers view AI-based systems and their outcomes as transparent and explainable. One manager clarifies that " $\ldots$ it is incredibly important that there is transparency. Yоu must be able to understand absolutely how a decision was made." (group 3). This criterium is underlined by the opinion that "... in today's world there is far too much manipulation." (group 3). Finally, expert availability is also important, because "It's still easiest to pick up the phone because you hope that the person you're talking to will make the selection for you." (group 2). The managers also communicated barriers to AI usage in decision-making: “... it is human nature to trust a human contact more than a machine. Even if it is fully transparent." (group 1). Others state that "Just because there is a neural network here now, for example ... so we put data in the front and the result comes out in the back. Then I would say: Well, I would rather not. If I now know that there is a decision tree behind it, which decides one way or another, depending on where and at which node which decision is made, then it can be clearly understood. And then trust is there." (group 1). In general, the managers currently put not as much trust in AI-based systems than in humans: "[...] for your work areas it is more likely that you trust the personal contact more due to the maturity of the technology." (group 2). On the other hand, managers are aware about the fact that AIbased systems are often more accurate than humans: "[...] you are always harsher with technology than with people, right? I am thinking about autonomous driving. I mean, if AI had an accident rate that was close to that of individuals and persons, then the AI would not get very far. [...] just one or two accidents could mean the end to autonomous driving. But the fact that we have had millions of accidents ourselves, you kind of block that out." (group 2). Even though managers fear AI that develops its own agenda one day, they clarify that they generally believe in the technology, especially with growing maturity: "As soon as it becomes clear how AI derives the individual results and outputs, people will trust it." (group 2). Moreover, the precision of the recommendations generated by an AI-based tool may be a barrier in managerial decision-making: "I keep thinking how much Amazon, for example, knows about me, I am always amazed how bad the predictions are and what is suggested to me." (group 1). The participants also identify advantages of AI related to decision-making velocity, preparation, and the exclusion of emotionality. "In fraud detection, [...] AI is simply much faster. And you can process much more data than a human being." (group 3). Managers also see advantage in "... AI making a preliminary decision, a pre-selection, but the final decision, an action, is made by the human being." (group 3). Fourth and lastly, the managers fear the disadvantages of AI application, namely a lack of human component, potential misuse of the technology and its difficulties to deal with irrational situations: “... when it comes to relevant decisions, [...] personal knowledge, or at least the feeling of personal knowledge, plays an enormous role ...” (group 3). The last statement reveals an interesting contradiction to the exclusion of emotionality which the participants view as an AI advantage. While managers fear emotionality in decision-making and view it as an advantage that AI can exclude feelings, they fear to lose the human component of their work. Possible misuse because of the black box-character of some AI systems also plays a role: “... every person has a 'hidden agenda'. And maybe decisions will be made based on this agenda. This means that I actually trust the human being more than the black box AI only because I know that it is a human being. This sounds a bit absurd, but it boils down to this, doesn't it?" (group 3). Finally, the managers consider AI to cause difficulties in irrational decision-making situations: "I believe that these intuitive decisions never go away completely because, I would say, you talk about this issue in-house, mostly with other people." (group 3).

\section{Validation of focus group themes}

A validation survey was conducted with 31 bank, insurance and bank consulting managers from Germany, Austria, and Switzerland which are disjunct from the focus group participants. Part 1 and 2 of the survey statements are based on the themes identified during the focus group interviews, part 3 aims to identify human-AI decision-making structures as well as potential tasks and roles that managers would delegate/assign to AI systems. The stand-alone, 
Likert-type statements are presented in Table 3 together with the rating distribution on a five-point Likert scale that was chosen due to the time constraints of the participants. Compared to a seven-point Likert scale, which is considered more precise, the five-point Likert scale has the advantage that it can be understood more quickly by participants which was crucial in the survey due to their time constraints [43]. Overall, 26 of the 37 statements were confirmed by the managers. Due to space limitations, the analysis focuses on neutral and refused statements. The availability of experts seems to have a limited influence on the use of AI-based systems in decision-making (5.4). If experts are on site, they will be consulted; otherwise, the AI system is consulted - rapid availability seems to be the primary factor. Additionally, managers' trust in AI technology does not appear to be an obstacle to its use in decision-making (6.1) and there is little doubt about the AI accuracy (6.2). Furthermore, the managers are not classifying human absence as a negative aspect (8.1) and information misuse is only feared to a limited extent (8.2). As expected, 55\% of managers cannot imagine a complete task delegation to an AI system (9.1), likewise no AI-based decision based on the managers' suggestion is desired (9.3). However, 71\% of the participants would welcome decision proposals from intelligent applications (9.2) or would consider aggregated decision-making (9.3). The statements on the delegation of tasks to AI systems (10) are largely agreed with, but managers are reluctant to implement decisions. Furthermore, they can imagine decisionmaking roles as well as interpersonal, and informational roles for $\mathrm{AI}$ in their organizations (11).

\section{Discussion}

The analysis results regarding RQ1 indicate that IP and the ever-increasing IO are important issues for the integration of AI into managerial decision-making. Managers are spending more and more time on information acquisition and synthesis to construct meaning from the information. IO manifests itself not only in information acquisition due to unstructured information and a lack of appropriate IP tools. The study results also imply negative effects on the managers' information synthesis, as relevance and objectivity are difficult to assess due to the amount of information. Additionally, managers proactively think about IS usage and repeatedly communicate implicit criteria for their use in IP. They are aware of the

Table 3. Validation study results including the rating distribution on a five-point Likert scale.

\begin{tabular}{|c|c|c|c|c|c|c|c|}
\hline \multirow{19}{*}{ 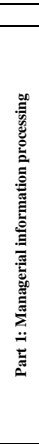 } & & Statements & \multicolumn{5}{|c|}{ Likert item rating distribution } \\
\hline & 1 & Information processing challenges & [1] & [2] & [3] & [4] & [5] \\
\hline & 1.1 & The amount of information to be processed in my job is constantly increasing. & $0,00 \%$ & $0,00 \%$ & $3,23 \%$ & $22,58 \%$ & $74,19 \%$ \\
\hline & 1.2 & The relevance assessment of incoming information takes more and more time. & $0,00 \%$ & $3,23 \%$ & $6,45 \%$ & $41,94 \%$ & $48,39 \%$ \\
\hline & 1.3 & The objectivity assessment of incoming information takes more and more time. & $0,00 \%$ & $9,68 \%$ & $16,13 \%$ & $51,61 \%$ & $22,58 \%$ \\
\hline & 2 & Information overload & & & & & \\
\hline & 2.1 & I receive a lot of unstructured information. & $0,00 \%$ & $3,23 \%$ & $9,68 \%$ & $70,97 \%$ & $16,13 \%$ \\
\hline & 2.2 & I do not have the time to process the mass of information. & $0,00 \%$ & $3,23 \%$ & $22,58 \%$ & $48,39 \%$ & $25,81 \%$ \\
\hline & 2.3 & I lack tools to support me in processing all the information I receive daily. & $0,00 \%$ & $12,90 \%$ & $22,58 \%$ & $38,71 \%$ & $25,81 \%$ \\
\hline & 2.4 & I feel overwhelmed by the amount of information I get on the internet when I research subjects there. & $0,00 \%$ & $32,26 \%$ & $29,03 \%$ & $29,03 \%$ & $9,68 \%$ \\
\hline & 3 & Information system usage & & & & & \\
\hline & 3.1 & My use of information systems depends on the nature of the search, e.g., the time pressure and the effort of the search. & $0,00 \%$ & $12,90 \%$ & $3,23 \%$ & $51,61 \%$ & $32,26 \%$ \\
\hline & 3.2 & $\ldots$ depends on the decision context, e.g., whether it is a credit decision or a strategic decision. & $0,00 \%$ & $12,90 \%$ & $3,23 \%$ & $54,84 \%$ & $29,03 \%$ \\
\hline & 3.3 & $\ldots$ depends on my experience in the application domain. & $0,00 \%$ & $6,45 \%$ & $16,13 \%$ & $48,39 \%$ & $29,03 \%$ \\
\hline & 3.4 & $\ldots$ depends on the type of problem to be solved, e.g., whether it is a reasonably well-known problem or a completely new one. & $0,00 \%$ & $3,23 \%$ & $3,23 \%$ & $48,39 \%$ & $45,16 \%$ \\
\hline & 4 & Information system integration & & & & & \\
\hline & 4.1 & The internal data structure is hindering information system integration of my organization. & $0,00 \%$ & $16,13 \%$ & $12,90 \%$ & $51,61 \%$ & $19,35 \%$ \\
\hline & 4.2 & Privacy regulations are hindering information system integration of my organization. & $0,00 \%$ & $16,13 \%$ & $12,90 \%$ & $48,39 \%$ & $22,58 \%$ \\
\hline & 4.3 & A lack of process integration is hindering information system integration of my organization. & $3,23 \%$ & $12,90 \%$ & $9,68 \%$ & $45,16 \%$ & $29,03 \%$ \\
\hline \multirow{16}{*}{ 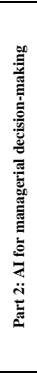 } & 5 & AI usage for managerial decision-making & & & & & \\
\hline & 5.1 & My use of AI depends on having a solid database. & $3,23 \%$ & $6,45 \%$ & $19,35 \%$ & $38,71 \%$ & $32,26 \%$ \\
\hline & 5.2 & $\ldots$ depends on whether the results can be validated by a human. & $3,23 \%$ & $29,03 \%$ & $19,35 \%$ & $32,26 \%$ & $16,13 \%$ \\
\hline & 5.3 & $\ldots$ depends on whether the results are so transparent that I can understand them independently. & $6,45 \%$ & $6,45 \%$ & $12,90 \%$ & $41,94 \%$ & $32,26 \%$ \\
\hline & 5.4 & $\ldots$ depends on whether experts are available to support me in decision-making. & $6,45 \%$ & $9,68 \%$ & $45,16 \%$ & $32,26 \%$ & $6,45 \%$ \\
\hline & 6 & Barriers for AI usage in managerial decision-making & & & & & \\
\hline & 6.1 & I generally lack trust in AI-based systems. & $38,71 \%$ & $45,16 \%$ & $9,68 \%$ & $6,45 \%$ & $0,00 \%$ \\
\hline & 6.2 & I have doubts about the accuracy of the results of AI-based systems. & $19,35 \%$ & $41,94 \%$ & $22,58 \%$ & $16,13 \%$ & $0,00 \%$ \\
\hline & 7 & Advantages of AI usage in managerial decision-making & & & & & \\
\hline & 7.1 & AI allows me to make decisions quicker than without using it. & $3,23 \%$ & $3,23 \%$ & $19,35 \%$ & $61,29 \%$ & $12,90 \%$ \\
\hline & 7.2 & AI supports me in the preparation of decisions. & $6,45 \%$ & $3,23 \%$ & $6.45 \%$ & $61,29 \%$ & $22,58 \%$ \\
\hline & 7.3 & AI excludes emotional influences in decision-making. & $6,45 \%$ & $19,35 \%$ & $16,13 \%$ & $41,94 \%$ & $16,13 \%$ \\
\hline & 8 & Disadvantages of AI usage in managerial decision-making & & & & & \\
\hline & 8.1 & With AI-assisted decisions, I miss the human component. & $9,68 \%$ & $35,48 \%$ & $32,26 \%$ & $19,35 \%$ & $3,23 \%$ \\
\hline & 8.2 & $\ldots, \mathrm{I}$ am afraid of misuse of the processed information or the results. & $9,68 \%$ & $32,26 \%$ & $25,81 \%$ & $29,03 \%$ & $3,23 \%$ \\
\hline & 8.3 & ..., I worry that the systems will misclassify irrational situations. & $6,45 \%$ & $25,81 \%$ & $22,58 \%$ & $38,71 \%$ & $6,45 \%$ \\
\hline \multirow{14}{*}{ 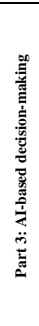 } & 9 & Human-AI decision-making structure & & & & & \\
\hline & 9.1 & I prefer the complete delegation of my decisions to an AI-based system. & $32,26 \%$ & $54,84 \%$ & $9,68 \%$ & $3,23 \%$ & $0,00 \%$ \\
\hline & 9.2 & ... a suggestion of the Al-based system, which I can accept or reject. & $0,00 \%$ & $3,23 \%$ & $0,00 \%$ & $70,97 \%$ & $25,81 \%$ \\
\hline & 9.3 & ... to make a suggestion to the AI-based system, which can be accepted or rejected by the system. & $9,68 \%$ & $48,39 \%$ & $22,58 \%$ & $19,35 \%$ & $0,00 \%$ \\
\hline & 9.4 & $\ldots$ a form of human-AI decision-making in which the two decisions made separately by me and the system are aggregated according to a specific rule. & $0,00 \%$ & $16,13 \%$ & $35,48 \%$ & $35,48 \%$ & $12,90 \%$ \\
\hline & 10 & Task delegation to AI-based systems & & & & & \\
\hline & 10.1 & I would like an AI-based system to identify and point out problems. & $0,00 \%$ & $0,00 \%$ & $3,23 \%$ & $51,61 \%$ & $45,16 \%$ \\
\hline & 10.2 & $\ldots$ to propose courses of action. & $0,00 \%$ & $0,00 \%$ & $3,23 \%$ & $45,16 \%$ & $51,61 \%$ \\
\hline & 10.3 & $\ldots$ to choose the best of those option(s) for me, which I have worked out. & $3,23 \%$ & $12,90 \%$ & $22,58 \%$ & $45,16 \%$ & $16,13 \%$ \\
\hline & 10.4 & $\ldots$ to implement the decisions, I have made. & $0,00 \%$ & $25,81 \%$ & $25,81 \%$ & $32,26 \%$ & $16,13 \%$ \\
\hline & 11 & Roles for AI-based systems & & & & & \\
\hline & 11.1 & Decision-making role: I would like the AI-based system to give me recommendations for or against a defined course of action. & $0,00 \%$ & $3,23 \%$ & $0,00 \%$ & $61,29 \%$ & $35,48 \%$ \\
\hline & 11.2 & Interpersonal role: I would like the AI-based system to give me recommendations on how to proceed during the decision-making process. & $0,00 \%$ & $3,23 \%$ & $25,81 \%$ & $45,16 \%$ & $25,81 \%$ \\
\hline & 11.3 & Informational role: I want the AI-based system to provide me with additional information for the decision-making process. & $0,00 \%$ & $0,00 \%$ & $6,45 \%$ & $48,39 \%$ & $45,16 \%$ \\
\hline
\end{tabular}


technological barriers to IS integration in IP, e.g., due to inadequate data structures, privacy regulations, or lack of process integration. Financial industry managers seem to have general need for IP support, regardless of expert availability. The interview participants also formulate themes that contribute to the AI design for managerial decision-making. They mention the database, human validation, transparency, and expert availability as criteria for assessing the usefulness of AI in decision-making. Lack of trust and recommendation precision are seen as barriers to AI usage. From the managers' point of view, the increased speed of decision-making, the support in decision preparation and the exclusion of emotionality are advantages of AI. The lack of human component, possible misuse, and difficulties with irrational situations are seen as disadvantages of the technology.

The organizational implications regarding RQ2 arise under the impression of the managers' IO, whereby the study participants find AI usage beneficial and have few risk concerns. The study results underline that managers' IP could negatively influence managerial decision-making quality and pose consequences for the entire organization [25]. If the management cannot acquire, synthesize and make sense of information to generate high-quality insights for ill-defined problems, the derived organizational procedures and processes will not provide long-term benefits [29] [30]. The study participants appear to be aware of the risks and value of AI functionalities and are open to AI-based IP and decision-making support. Nevertheless, the managers refuse to fully transfer decision-making authority to intelligent systems due to remaining trust issues, even though they are aware that human decisions can also be 'black boxes' since humans make partially intuitive decisions. Instead, managers prefer AI-to-human sequential decisionmaking which offers a pre-selection of options according to defined criteria for humans, good interpretability of results due to human involvement, and a large set of alternative sets due to AI involvement, which is advantageous and important for usage in the highly regulated financial industry. On the other hand, AI may incorrectly discard options, the replicability of the decision process is low due to human variability, and the speed of decision-making is relatively slow [9]. The before mentioned findings not only extend knowledge on the symbiosis of human and AI from a managerial perspective, but also underline negative aspects of AI-based leadership elaborated by Parry et al. (2016) [4] [6]. Furthermore, the present study shows that managers value the advantages of hybrid intelligence which combines both intuitive and analytical strengths of humans and AI [3]. All in all, the study findings call companies to evaluate the mentioned positive and negative aspects of AI integration for management decision-making, as they have spillover effects on the entire organization, especially in the case of ill-defined problems. Concerning well-defined problems, AI system integration, e.g., for filtering information could be a first step to relieve managers of IO, improve their IP behavior and sustain decision quality at a high level.

\section{Conclusion}

The present study analyzes relevant issues for AI integration into managerial decision-making and develops organizational implications. The results highlight the link between managerial IP and decisionmaking and expand knowledge about the need for and design of AI support. IO negatively affects managerial information acquisition and synthesis, which lack tool support. To empower managerial IP and decisionmaking, organizations must (1) evaluate managerial IP tasks and matching AI functionalities and systems, (2) (re)define roles for managers and AI systems, and (3) redesign management processes for sustainable human-AI interaction. A collaborative improvement cycle involving technical experts and subject matter experts should be established to prevent misuse of AI systems and avoid management and workforce mistrust in the technology should failures occur.

\section{References}

[1] Reinsel, D., J. Gantz, and J. Rydning, "The Digitization of the World", IDC White Paper No. US44413318., 2020, pp. 24.

[2] Pisarchik, A.N., V.A. Maksimenko, and A.E. Hramov, "From Novel Technology to Novel Applications: Comment on 'An Integrated Brain-Machine Interface Platform With Thousands of Channels' by Elon Musk and Neuralink", Journal of Medical Internet Research 21(10), 2019, pp. e16356.

[3] Dellermann, D., P. Ebel, M. Söllner, and J.M. Leimeister, "Hybrid Intelligence", Business \& Information Systems Engineering 61(5), 2019, pp. 637-643.

[4] Jarrahi, M.H., "Artificial intelligence and the future of work: Human-AI symbiosis in organizational decision making", Business Horizons 61(4), 2018, pp. 577-586.

[5] Zheng, N., Z. Liu, P. Ren, et al., "Hybrid-augmented intelligence: collaboration and cognition", Frontiers of Information Technology \& Electronic Engineering 18(2), 2017, pp. 153-179.

[6] Parry, K., M. Cohen, and S. Bhattacharya, "Rise of the Machines: A Critical Consideration of Automated Leadership Decision Making in Organizations", Group \& Organization Management 41(5), 2016, pp. 571-594.

[7] Duan, Y., J.S. Edwards, and Y.K. Dwivedi, "Artificial intelligence for decision making in the era of Big Data - evolution, challenges and research agenda", 
International Journal of Information Management 48, 2019, pp. 63-71.

[8] von Krogh, G., "Artificial Intelligence in Organizations: New Opportunities for Phenomenon-Based Theorizing", Academy of Management Discoveries 4(4), 2018, pp. 404-409.

[9] Shrestha, Y.R., S.M. Ben-Menahem, and G. von Krogh, "Organizational decision-making structures in the age of artificial intelligence", California Management Review 61(4), 2019, pp. 66-83.

[10] Seidel, M.-D.L., "Questioning Centralized Organizations in a Time of Distributed Trust.”, Journal of Management Inquiry 27(1), 2018, pp. 40-44.

[11] Duan, Y., V.K. Ong, M. Xu, and B. Mathews, "Supporting decision making process with 'ideal' software agents - What do business executives want?", Expert Systems with Applications 39(5), 2012, pp. 5534-5547.

[12] Simon, H.A., The New Science of Management Decision, Prentice Hall, NJ, 1977.

[13] Ong, V.K., Y. Duan, and M. Xu, "Senior Managers' Information Behavior in Current Emerging Ubiquitous and Intelligent Computing Environment", Proceedings of the 53rd Hawaii International Conference on System Sciences, (2020), pp. 5952-5961.

[14] Bawden, D., and L. Robinson, "No such thing as society? On the individuality of information behavior", Journal of the American Society for Information Science and Technology 64(12), 2013, pp. 2587-2590.

[15] Wilson, T.D., "Models in information behaviour research", Journal of Documentation 55(3), 1999, pp. 249-270.

[16] Wilson, T.D., "On User Studies and Information Needs", Journal of Documentation 37(1), 1981, pp. 315.

[17] Ellis, D., D. Cox, and K. Hall, "A Comparison of the Information Seeking Patterns of Researchers in the Physical and Social Sciences", Journal of Documentation 49(4), 1993, pp. 356-369.

[18] Kuhlthau, C.C., "A principle of uncertainty for information seeking”, Journal of Documentation 49(4), 1993, pp. 339-355.

[19] Wilson, T.D., "Information behaviour: An interdisciplinary perspective", Information Processing \& Management 33(4), 1997, pp. 551-572.

[20] Spink, A., Information Behavior: An Evolutionary Instinct, Springer Science \& Business Media, 2010.

[21] Hiltz, S.R., and M. Turoff, "Structuring computermediated communication systems to avoid information overload", Communications of the ACM 28(7), 1985, pp. 680-689.

[22] Roetzel, P.G., "Information overload in the information age: a review of the literature from business administration, business psychology, and related disciplines with a bibliometric approach and framework development", Business Research 12(2), 2019, pp. 479522.

[23] Eppler, M.J., and J. Mengis, "The Concept of Information Overload: A Review of Literature from Organization Science, Accounting, Marketing, MIS, and Related Disciplines", The Information Society 20(5), 2004, pp. 325-344.

[24] Schroder, H.M., M.J. Driver, and S. Streufert, Human information processing: individuals and groups functioning in complex social situations, Holt, Rinehart and Winston, New York, 1967.

[25] Miller, J.G., "Information input overload and psychopathology", American Journal of Psychiatry 116(8), 1960, pp. 695-704.

[26] Meier, R.L., "Information Input Overload: Communications-Oriented Institutions", Libri 13, 1963, pp. 1.

[27] Ackoff, R.L., "Management Misinformation Systems", Management Science 14(4), 1967, pp. B-147.

[28] Sobotta, N., "A Systematic Literature Review on the Relation of Information Technology and Information Overload", 2016 49th Hawaii International Conference on System Sciences (HICSS), IEEE (2016), pp. 858867.

[29] Soelberg, P., "Unprogrammed Decision Making.", Academy of Management Proceedings, Academy of Management (1966), pp. 3-16.

[30] Simon, H.A., "The Structure of Ill Structured P coblems", Artificial Intelligence 4(3-4), 1973, pp. 181201.

[31] Edmunds, A., and A. Morris, "The problem of information overload in business organisations: a review of the literature", International Journal of Information Management 20(1), 2000, pp. 17-28.

[32] Mintzberg, H., Structure in fives: Designing effective organizations, Prentice-Hall, Inc, Englewood Cliffs, NJ, US, 1992.

[33] Mintzberg, H., Tracking strategies: towards a general theory of strategy formation, Oxford University Press, New York, NY, 2009.

[34] Simon, H.A., The new science of management decision, Harper, New York, 1960.

[35] Agrawal, A., Gans, J., Goldfarb, A., Prediction Machines: The Simple Economics of Artificial Intelligence, Harvard Business Press, 2018.

[36] Dietzmann, C., and R. Alt, "Assessing the Business Impact of Artificial Intelligence", Proceedings of the 53rd Hawaii International Conference on System Sciences., (2020), pp. 5170-5179.

[37] Gardner, H.E., Intelligence Reframed: Multiple Intelligences for the 21st Century, Hachette UK, 2000.

[38] Russell, S., and P. Norvig, Artificial Intelligence: A Modern Approach, Malaysia, 2016.

[39] Frantz, R., "Herbert Simon. Artificial intelligence as a framework for understanding intuition", Journal of Economic Psychology 24(2), 2003, pp. 265-277.

[40] Alt, R., R. Beck, and M.T. Smits, "FinTech and the transformation of the financial industry", Electronic Markets 28(3), 2018, pp. 235-243.

[41] Mason, J., Qualitative Researching, Sage, 2017.

[42] Morse, J.M., “'Perfectly Healthy, but Dead': The Myth of Inter-Rater Reliability", Qualitative Health Research 7(4), 1997, pp. 445-447.

[43] Joshi, A., S. Kale, S. Chandel, and D. Pal, "Likert Scale: Explored and Explained", British Journal of Applied Science \& Technology 7(4), 2015, pp. 396-403. 